\title{
What are the limits to cell plasticity?
}

\author{
Jane Taylor ${ }^{1}$, Ian Wilmut ${ }^{1}$, Gareth Sullivan ${ }^{1}$ \\ ${ }^{1}$ MRC Centre for Regenerative Medicine, University of Edinburgh, GU.507 Chancellor's Building, 49 Little France Crescent, \\ Edinburgh, EH16 4SB, UK \\ Cell Research (2010) 20:502-503. doi:10.1038/cr.2010.59; published online 3 May 2010
}

It is now well established that the fate of a somatic cell is not fixed rigidly and that there is a significant degree of cell plasticity. The term plasticity refers to the opportunity to change differentiated cells from one cell type to another. Over the past 25 years a series of papers have each demonstrated that plasticity is wider than had previously been understood [1-4]. An exciting recent article by Thomas Vierbuchen and colleagues at Stanford University extended that series by describing a method for directly reprogramming mouse fibroblast cells into neurons without the need to generate a stem cell intermediary [5].

Vierbuchen and colleagues isolated fibroblasts from embryos (mouse embryonic fibroblasts - MEFs) and postnatal tail-tips from transgenic (TauEGFP knock-in) mice. TauEGFP reporter mice express the green fluorescent protein in post-mitotic neurons thus allowing visualisation of motor and sensory neuronal cells as they develop, aiding the identification of successful reprogramming. A drug-inducible lentiviral system containing 19 candidate genes selected as being either expressed in neural cell types or essential for reprogramming to pluripotency was used to reprogramme fibroblast to a neuronal state. From this group five factors that were able to evoke a neuronal phenotype were Brn2, Myt1l, Zic1, Olig2 and

Correspondence: Ian Wilmut

E-mail: ian.wilmut@ed.ac.uk
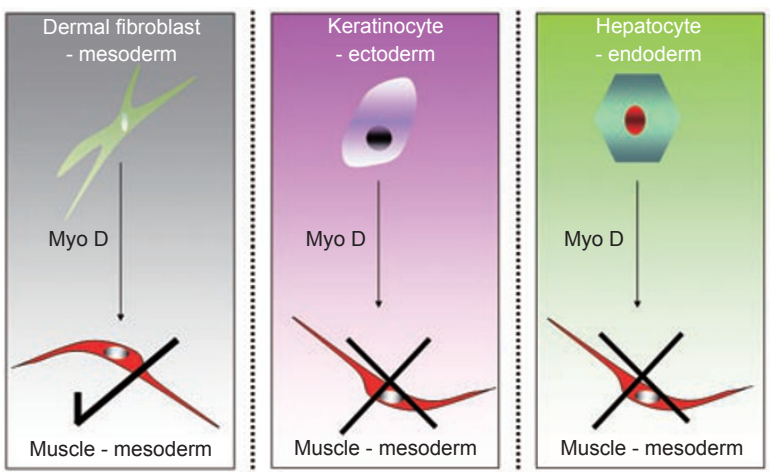

Figure 1 Restricted lineage reprogramming. An example of restricted transdifferentiation is when the transcription factor MyoD is ectopically expressed in dermal fibroblasts (Mesoderm) converting them to muscle (Mesoderm). When this experiment was repeated in other lineages, namely Ectoderm or Endoderm, no conversion to muscle was observed but activation of some muscle specific genes was observed. This observation is not an isolated case, the conversion of exocrine cell within the pancreas to an endocrine phenotype by the expression of three factors (not shown) [10]. Therefore there is a necessity to demonstrate unequivocally the conversion of one lineage to another utilising defined cell types.

Ascll. They termed the reprogrammed cells induced neuronal (iN) cells. The findings demonstrate MEF-derived iN cells (i) express neuron-specific markers including Tuj-1, MAP2, NeuN, Synapsin and GABA and (ii) exhibit functional neuronal properties including the generation of action potentials and formation of functional synapses. Similar results were obtained when postnatal tail-tip fibroblast (TTF) cells were reprogrammed. By sequentially reducing the number of transcription factors Vierbuchen et al. [5] identified a combination of just three genes, Brn2, Myt1l, and Ascl1, that were capable of inducing neurons from TTF and MEFs. Neuronal induction was both fast and efficient in comparison to reprogramming to pluripotent cells [1] with cells exhibiting an immature neuron-like morphology as early as 3 days post-infection and reprogramming efficiency rates of up to $19.5 \%$. In light of this high efficiency, it is very unlikely that the observations are the result of directed differentiation of rare stem or precursor cells.

The demonstration of both rapid and efficient conversion of fibroblasts to a population of predominantly excitatory cortical neurons reaffirms that somatic cell populations are not fixed and indeed 
have huge plasticity. This observation is particularly exciting because of the possibility that the change was between two different lineages, mesoderm to ectoderm, although this is not discussed in detail by the authors in the original article itself [5]. There is a degree of uncertainty because the starting material was a heterogeneous cell population, probably containing both mesoderm and ectoderm. Thus, the resulting iN cells may have been derived from a population within the same lineage i.e. ectoderm.

The current literature landscape suggested that change is restricted to within the same lineage when employing forced expression of transcription factors. For example (see Figure 1), expression of MyoD converts fibroblasts (mesoderm) to myotubes (mesoderm) [6] whilst this conversion is not observed in hepatocytes (endoderm) or keratinocytes (ectoderm) [7, 8]. One could speculate that this is a consequence of plasticity within that tissue lineage itself. The issue of trans-differentiation between lineages (inter-) will only be resolved as and when a defined cell population such as CD45+ cells from blood (mesoderm) is successfully re-assigned to another cellular identity for example a neuron (ectoderm). While some change may be directed by transcription factors alone, typically the process is slow and inefficient as it requires more than a week and changes only a small proportion of the cells. By contrast, somatic cell nuclear transfer and cell fusion bring about change more efficiently and within 24 and $48 \mathrm{~h}$, respectively [2,9]. It is not clear whether this difference reflects the influence of other factors or the presence of a greater concentration of transcription factors within the nucleus. It remains to be shown whether or not transcription factors alone are able to direct change between lineages or will require the involvement of other factors.

The experiments described by Vierbuchen $e t$ al., gave rise by default to a specific population of neurons. The authors speculate that expression of specific subsets of factors as yet not identified may generate populations of multipotent progenitors, such as neural stem cells, which in turn could be efficiently patterned to multiple neuronal cell types. The use of integrative lentivirus will limit its application for therapeutic approaches. However, as with the iPS approaches, there is a concerted effort to eliminate the use of genome alteration by utilising alternative methods such as employing small molecules to mimic the effect of the transcription factors. These in turn could be translated to this model system.

Therefore the ability to translate this methodology directly to the human situation by converting donor somatic tissue of a defined identity, to a cell type afflicted with a cellular degeneration would benefit both modelling of human disease and aid drug discovery. In addition, this approach is not restricted to the generation of neurons but could potentially be applied to generate other cell types as and when the key transcriptional cues are determined. To fully realise this technology, a concerted effort will be required to demonstrate the ability to accurately induce alteration of lineage fate as to reflect the in vivo cellular equivalent!

\section{References}

1 Wilmut I, Schnieke AE, McWhir J, Kind
AJ, Campbell KH. Viable offspring derived from fetal and adult mammalian cells. Nature 1997; 385:810-813.

2 Tada M, Takahama Y, Abe K, Nakatsuji $\mathrm{N}$, Tada T. Nuclear reprogramming of somatic cells by in vitro hybridization with ES cells. Curr Biol 2001; 11:15531558.

3 Håkelien AM, Landsverk HB, Robl JM, Skålhegg BS, Collas P. Reprogramming fibroblasts to express T-cell functions using cell extracts. Nat Biotechnol 2002; 20:460-466.

4 Takahashi K, Yamanaka S. Induction of pluripotent stem cells from mouse embryonic and adult fibroblast cultures by defined factors. Cell 2006; 126:663676.

5 Vierbuchen T, Ostermeier A, Pang ZP, Kokubu Y, Südhof TC, Wernig M. Direct conversion of fibroblasts to functional neurons by defined factors. Nature 2010; 463:1035-1041.

6 Davis R, Weintraub H, Lassar AB. Expression of a single transfected cDNA converts fibroblasts to myoblasts. Cell 1987; 51:987-1000.

7 Schäfer BW, Blakely BT, Darlington GJ, Blau HM. Effect of cell history on response to helix-loop-helix family of myogenic regulators. Nature 1990; 344:454-458.

8 Boukamp P, Chen J, Gonzales F, Jones PA, Fusenig NE. Progressive stages of "transdifferentiation" from epidermal to mesenchymal phenotype induced by MyoD1 transfection, 5-aza-2'deoxycytidine treatment, and selection for reduced cell attachment in the human keratinocyte line HaCaT. J Cell Biol 1992; 116:1257-1271.

9 Wakayama T, Perry AC, Zuccotti M, Johnson KR, Yanagimachi R. Full-term development of mice from enucleated oocytes injected with cumulus cell nuclei. Nature 1998; 394:369-374.

10 Zhou Q, Brown J, Kanarek A, Rajagopal J, Melton DA. In vivo reprogramming of adult pancreatic exocrine cells to betacells. Nature 2008; 455:627-632. 